\title{
Regarding the functional equivalence of response-independent events and extinction
}

\author{
ROBERT L. WELKER and KATHLEEN MCAULEY \\ Mount Holyoke College, South Hadley, Massachusetts 01075
}

\begin{abstract}
Rats were trained to press a lever for food according to a variable-interval (VI) 60-sec schedule of reinforcement. They were then divided into four groups and received 10 sessions of either extinction or response-independent pellets delivered according to a variable-time (VT) $30-, 60-$, or 120 -sec schedule. Leverpressing was reduced at approximately the same rate for subjects receiving extinction, VT 30 - and VT 120 -sec pellet deliveries. Relative to thse groups, the VT 60-sec group exhibited slower reductions in responding. Subjects in the three VT groups were most frequently observed to contact the food magazine, whereas, subjects in the extinction group typically faced away from the magazine and appeared to be sleeping.
\end{abstract}

Extinction of operant behavior is functionally defined by two conditions: removal of the reinforcer and reduction in the frequency of responding. The conditions of the definition are relatively simple. It is a complicated matter, however, to determine what variables are responsible for reductions in the frequency of responding when reinforcers are withheld. For example, removal of the reinforcing stimulus is accompanied by removal of the contingency or systematic relation between responding and delivery of the reinforcer. It has been demonstrated repeatedly that removal of the contingency between responding and the reinforcing stimulus without withdrawal of the reinforcer (response-independent schedule) will reduce the frequency of a previously established operant response (e.g., Boakes, 1973; Edwards, West, \& Jackson, 1968; Herrnstein, 1966; Lattal, 1972; Rescorla \& Skucy, 1969; Skinner, 1938). In all of these instances, however, response-independent schedules were less effective in eliminating responding than was removal of the reinforcer. The latter has consistently produced more rapid reductions in response rates and/or lower asymptotic levels of responding.

Some of the explanations that have been offered to account for the differential effectiveness of the two procedures are as follows: (1) superstitious maintenance of responding during the response-independent schedule because of accidental pairings of the response and reinforcer (Herrnstein, 1966), (2) presentations of the reinforcing stimulus during response-independent conditions promote accidental responding by keeping the animal in the proximity of the response manipulandum

This research was supported by a faculty grant from Mount Holyoke College and National Institute of Mental Health Grant 1 RO3 MH28552-01 awarded to R. L. Welker. The authors thank Will Millard for statistical assistance. Requests for reprints should be sent to Robert L. Welker, Department of Psychology and Education, Mount Holyoke College, South Hadley, Massachusetts 01075. or by enhancing general activity (Rescorla \& Skucy, 1969), and (3) less generalization decrement in the transition from contingency to response-independent schedule than to extinction because the reinforcer is maintained in the former (Mackintosh, 1974).

Our experiment borrows from these explanations in addressing the variables that might be responsible for the greater effectiveness of extinction than responseindependent schedules in eliminating operant responding. In the studies cited above, subjects were first trained to respond for food according to a particular schedule of partial reinforcement (e.g., fixed interval, variable interval, or fixed ratio: FI, VI, or FR). Next, they received response-independent deliveries of the reinforcing stimulus according to the same temporal parameters as those used in training the operant response. For example, rats trained to leverpress for food with a VI 2-min schedule subsequently received responseindependent food deliveries according to a variable-time (VT) 2-min schedule (Rescorla \& Skucy, 1969). We reasoned on the basis of the generalization-decrement theory of extinction (Mackintosh, 1974), and evidence that animals may use time as a stimulus (Dews, 1962), that changing the temporal parameters for food deliveries between the conditions of response-contingent and response-independent pellet deliveries might make the transition more detectable to the subject and thereby enhance the effectiveness of the responseindependent procedure in reducing responding.

This reasoning is indirectly supported by an inverse relation between rate of responding and the frequency of response-independent food deliveries when they are superimposed upon an ongoing response-food contingency (Cole, 1973; Deluty, 1976; Edwards, Peek, \& Wolfe, 1970; Rachlin \& Baum, 1972; Zeiler, 1976). In this procedure, however, lower rates of responding accompanying the more frequent response-independent food deliveries may be a function of the subjects' spending large amounts of time consuming food. 
Lachter, Cole, and Schoenfeld (1971) report an experiment that is more directly related to our reasoning. Two pigeons were trained to peck a key for food according to a VI 60-sec schedule with six stimuli randomly alternated on the response key. Each of the stimuli was later associated with VT 5-, 15-, 30-, 60-, 120 -, or 240-sec response-independent food deliveries, each being presented for 10 successive grain deliveries within each of 30 sessions. The short VT values produced temporary increases in responding, whereas the longest VT values produced rapid reductions in responding.

The Lachter et al. (1971) experiment did not include the typical extinction condition (removal of the reinforcer) for comparison of response reduction with the various VT schedules, and the temporary increase in responding during the short VT values may have reflected local contrast effects, as both subjects experienced all VT values within each session.

We tested the hypothesis that changing the temporal parameters for food deliveries between responsecontingent and response-independent conditions would enhance reductions in the rate of responding with a between-groups design to eliminate local contrast effects. Rats were trained to respond for food according to a VI 60-sec schedule and then received VT 30, 60-, or 120 -sec response-independent food deliveries, or extinction. In addition, systematic observations were made of each subject's behavior other than leverpressing during exposure to the VT schedules and extinction. These observations were made in order to assess the possibility that differences in behaviors other than leverpressing might account for the differential effectiveness of the two procedures in eliminating responses.

\section{METHOD}

\section{Subjects}

Subjects were 36 experimentally naive, male, SpragueDawley rats maintained at $80 \%$ of their ad-lib weight.

\begin{abstract}
Apparatus
Three operant chambers $(19.5 \times 21 \times 23 \mathrm{~cm})$ constructed of transparent acrylic plastic and aluminum were enclosed in sound-attenuating hulls. In each chamber, the opening for a recessed food magazine $(4 \times 4.5 \times 3.5 \mathrm{~cm})$ was located $1 \mathrm{~cm}$ above the grid floor in the center of the magazine wall. A response lever $(1 \times 2.5 \times 2 \mathrm{~cm})$ requiring approximately $21 \mathrm{~g}$ of static force to operate was located $3 \mathrm{~cm}$ to the left of the food magazine and $4.5 \mathrm{~cm}$ above the floor. Each chamber was continuously illuminated by a $6-\mathrm{W}$ bulb $(120 \mathrm{~V})$ and white noise $\left(60 \mathrm{~dB}\right.$ re: $\left..0002 \mathrm{dynes} / \mathrm{cm}^{2}\right)$ was presented throughout each session. Noyes $45-\mathrm{mg}$ pellets were delivered via a Gerbrands Model D-1 dispenser, and events in the chambers were programmed and monitored through standard relay and solid state circuitry located in an adjoining room.
\end{abstract}

\section{Procedure}

Subjects were trained to leverpress for food and then received 10 baseline sessions (60-min duration) in which leverpressing was reinforced according to a VI 60 -sec schedule. They were then divided into four groups $(n=9)$ and received 10 sessions under one of the following conditions: response-independent pellets delivered according to a VT $30-, 60$-, or $120-\mathrm{sec}$ schedule, or extinction. The range of intervals between pellet deliveries for the VI and VT schedules was as follows: VT 30, 3-60 sec; VT $60,5-115 \mathrm{sec}$; and VT 120, 5-240 sec. The frequency of leverpressing was recorded in successive 5-min intervals throughout these sessions and transformed to percentages of the mean rates of responding computed for the final four baseline sessions for individual subjects.

Subjects in the response-independent groups received 60 pellets/session, with session durations being 30,60 , and $120 \mathrm{~min}$ for the VT $30-, 60$, and 120 -sec groups, respectively. Session duration for the extinction group was held constant at $60 \mathrm{~min}$.

During the final four baseline sessions and throughout the response-independent and extinction sessions, observations of the following behaviors were recorded with a 5-min timesampling procedure: contacting the food magazine, contacting the lever, facing the back wall (opposite magazine wall), apparent sleeping, rearing, and grooming.

Following Session 10 of extinction and response-independent food deliveries, conditions were reversed for two sessions, so that the VT 30 -, 60-, and 120-sec groups received extinction, and the extinction group received VT 60-sec pellet deliveries.

\section{RESULTS}

Two subjects in the VT 120 groups and one subject in the VT 60 group were eliminated from the experiment because of temporary apparatus malfunctions. Figures 1 and 2 summarize the leverpressing data for the 12 sessions by showing mean percentages of baseline
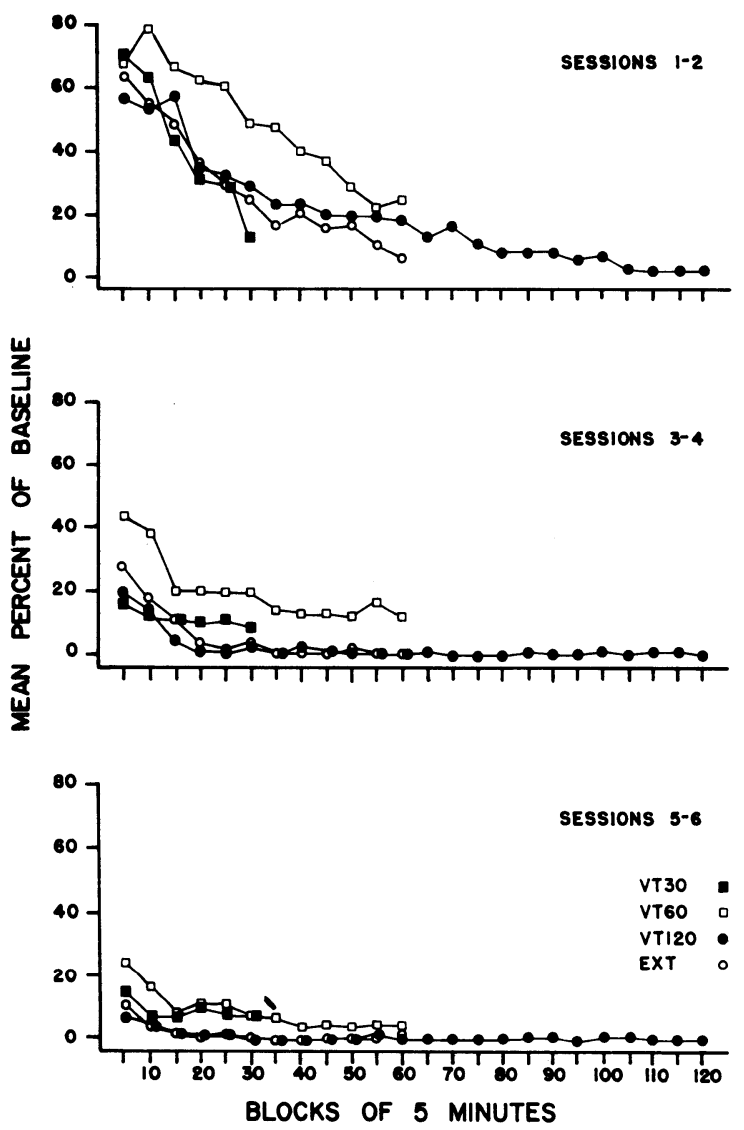

Figure 1. Mean percentages of baseline response rates during Sessions 1-6 of VT pellet deliveries and extinction. 

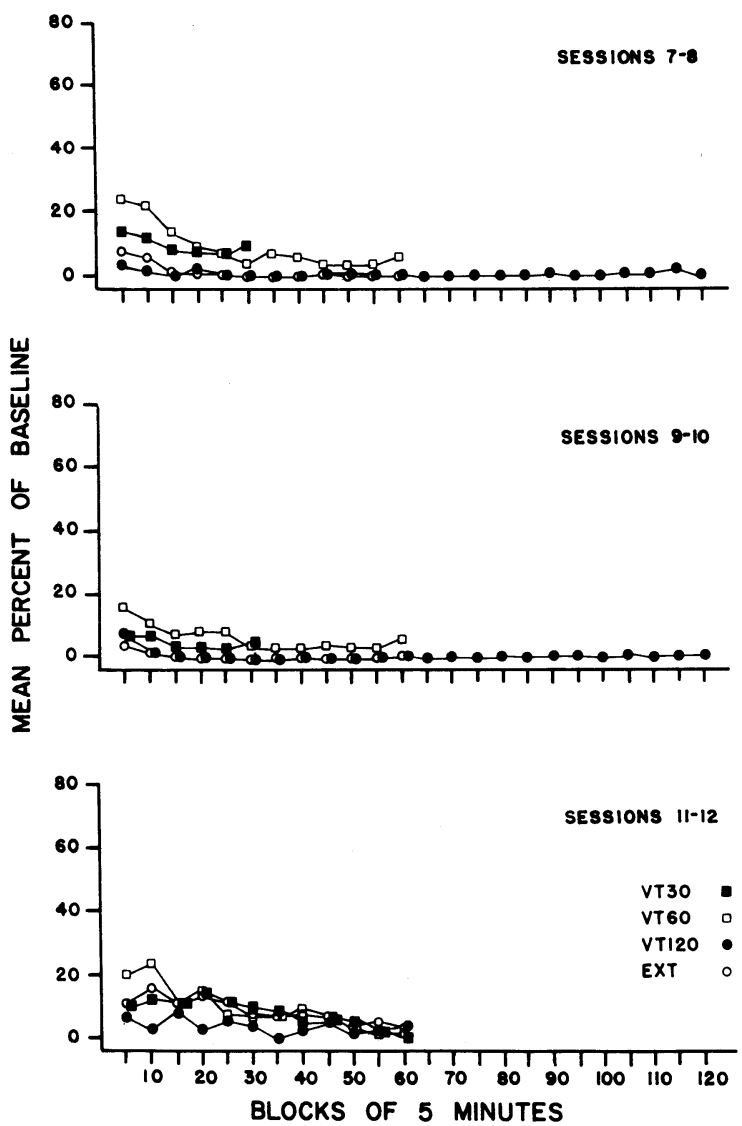

Figure 2. Mean percentages of baseline response rates during Sessions 7-12 of VT pellet deliveries and extinction.

responding during 5-min blocks of time within combined sets of 2 consecutive sessions. A mixed analysis of variance (groups by 5 -min blocks within sessions by combined sets of two sessions) was used to evaluate statistical significance of differences in leverpressing during Sessions 1-10. The analysis included only the first $30 \mathrm{~min}$ of each session, as this was the session duration for the VT 30-sec group.

As can be seen in Figures 1 and 2, all groups exhibited approximate negative decelerations in leverpressing as time elapsed within sessions [5-min blocks: $F(5,145)=$ $69.82, \mathrm{p}<.001]$, gradual reductions in leverpressing across sessions [sessions: $F(4,116)=119.40, p<.001$ ], and the VT 60 group generally responded at higher levels than the other three groups [groups: $F(3,29)=3.59$, $\mathrm{p}<.02]$. Duncan's Multiple Range tests applied to mean percentages of response measures for the 10 sessions combined indicated no statistically significant differences in responding for the VT 30, VT 120, and extinction groups, with each of these differing at the .05 level from the VT 60 group. The differences between the groups began to dissipate by Sessions 5-6, at which point the VT 60 group showed elevations in responding relative to the other groups only during the initial $5-10 \mathrm{~min}$ of the session [Groups by Minutes by Sessions: $F(60,580)=2.88, p<.001]$. Reversal of conditions during Sessions 11 and 12 produced no systematic effects upon leverpressing.

Figure 3 quantifies observations of contacting the food magazine, facing the back wall (facing away), and apparent sleeping. None of these behaviors differed among the four groups during baseline training, nor did the groups differ regarding observations of rearing, grooming, or contacting the lever during responseindependent feeding and extinction. In general, during Sessions 1-10, the subjects in all three VT groups were most frequently observed to contact the food magazine [groups: $F(3,29)=41.95, p<.001$ ], whereas subjects in the extinction group were most frequently observed to face away from the magazine wall [groups: $F(3,29)=$ $34.07, p<.001$ ] and sleep [groups: $F(3,29)=10.28$, $\mathrm{p}<.001]$. Frequency of observation of these behaviors was reversed for the VT and extinction groups following a reversal of conditions in Sessions 11 and 12.

\section{DISCUSSION}

The elevated response levels for subjects in the VT 60 group relative to the extinction group are consistent with several previous reports (Boakes, 1973; Edwards et al., 1968; Herrnstein,

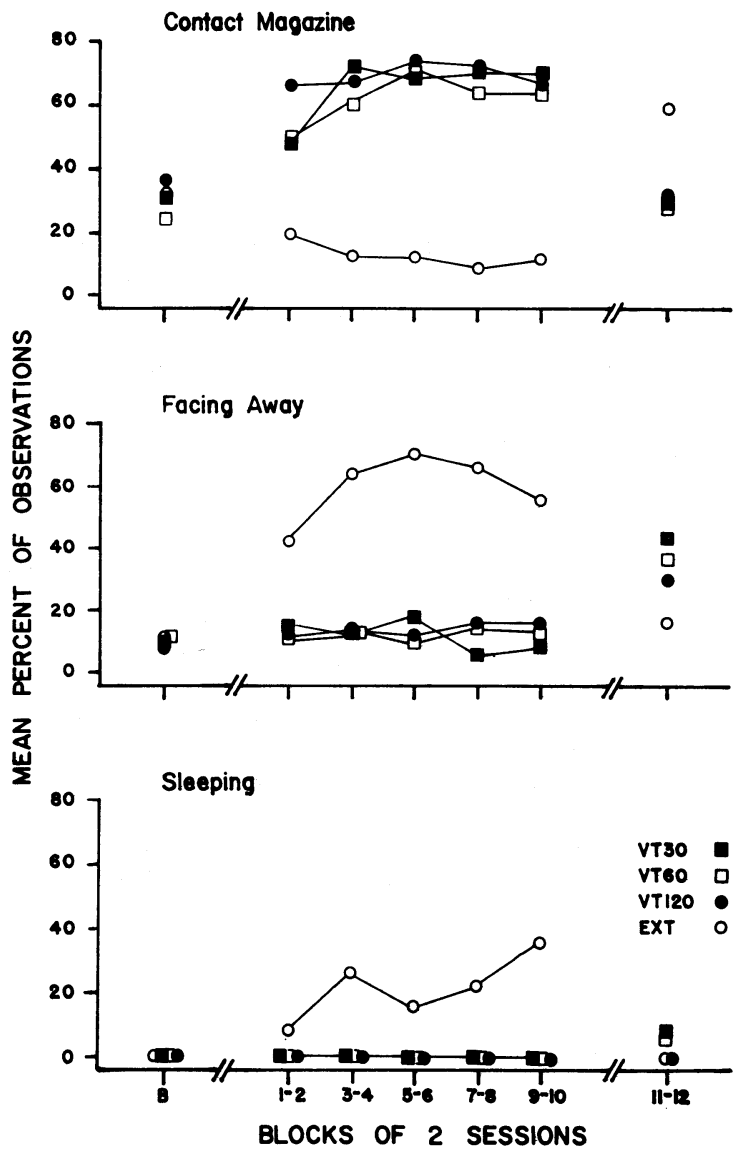

Figure 3. Observations of contacting food magazine (upper graph), facing the back wall (middle graph), and apparent sleeping (lower graph) during the 12 sessions of VT pellet deliveries and extinction. 
1966; Lattal, 1972; Rescorla \& Skucy, 1969). The roughly equivalent reductions in leverpressing for subjects in the VT 30 , VT 120, and extinction groups, however, demonstrate that when the interval between reinforcers is changed in the transition from response-contingent to response-independent conditions, the latter may be as effective as extinction in reducing rates of previously established operant responding. Therefore, with regard to this measure, the two procedures may be rendered functionally equivalent.

This functional equivalence breaks down, however, when behaviors other than the previously established operant response are considered. Subjects in the VT groups spent most of their time in contact with the food magazine, which is consistent with reports of the behavior of rats that are exposed to responseindependent feeding without previously receiving operant training (Davis \& Hubbard, 1972; Staddon \& Ayres, 1975). Subjects in the extinction group were most frequently observed to face away from the magazine wall and appeared to be sleeping. Although these behaviors served as a source of distinction between conditions of extinction and response-independent pellet deliveries, they did not distinguish between the three response-independent conditions that were differentially effective in reducing responding. This illustrates that the elevated response levels for the VT 60 group cannot simply be attributed to accidental responses that occurred because the responseindependent food deliveries kept the animal in the area of the response manipulandum.

Similar problems are encountered when attributing the elevated responding of the VT 60 group to accidental pairings of the response and food deliveries (i.e., superstition). This also fails to account for the differential reduction of responding among the VT $30-, 60$, and $120-$ sec groups. In fact, according to the position of Herrnstein (1966), superstitious maintenance of leverpressing should be strongest for the VT 30-sec group, a prediction not supported by our data.

Changing the interval between food deliveries for the VT 30 and VT 120 groups produced effects that are similar to changing contextual stimuli (e.g., ambient luminance and noise level, texture of floors, etc.) between conditions of acquisition and extinction (Welker \& McAuley, in press). This suggests that the average time between reinforcers may be processed as a cotextual stimulus by the subject, and maintenance of this contextual stimulus between response-contingent and responseindependent conditions may be responsible for reports of the differential effectiveness of response-independent schedules and extinction in eliminating previously established operant responding.

Finally, it should be noted that our VT 30,60 , and 120 groups were also differentiated by session duration during the response-independent conditions. We opted for equating the groups regarding the number of pellets per session rather than the amount of time per session, which would have resulted in differences in the number of pellets received by the subjects and problems in equating weights of subjects in the different groups. Although the absolute amount of time the subject spends in one condition will probably affect its behavior, the pattern of results depicted in Figures 1 and 2 was observed in the first $30 \mathrm{~min}$ of Session 1. This illustrates that the change in inter- reinforcer interval for the VT 30 and 120 groups was effective prior to any possible influence of the change in session duration.

\section{REFERENCES}

BoAKEs, R. A. Response decrements produced by extinction and by response-independent reinforcement. Journal of the Experimental Analysis of Behavior, 1973, 19, 293-302.

COLE, B. K. Schedules of concurrently contingent and non-contingent reinforcement. In F. J. McGuigan \& D. B. Lumsden (Eds.), Contemporary approaches to conditioning and learning. New York: Wiley, 1973.

Davis, H., \& HubBaRd, J. An analysis of superstitious behavior in the rat. Behaviour, 1972, 43, 1-12.

DEws, P. B. The effect of multiple $S$ periods on responding on a fixed-interval schedule. Journal of the Experimental Analysis of Behavior, 1962, 5, 369-374.

Deluty, M. Z. Excitatory and inhibitory effects of free reinforcers. Animal Learning \& Behavior, 1976, 4, 436-440.

Edwards, D. D., Peek, V., \& Wolfe, F. Independently delivered food decelerates fixed-ratio rates. Journal of the Experimental Analysis of Behavior, 1970, 14, 301-307.

EDw ARDS, D. D., West, J. P., \& JACKSON, V. The role of contingencies in the control of behavior. Psychonomic Science, $1968,10,39-40$.

HerRnstein, R. J. Superstition: A corollary of the principles of operant conditioning. In W. K. Honig (Ed.), Operant behavior: Areas of research and application. New York: Appleton-Century-Crofts, 1966.

Lachter, G. D., Cole, B. K., \& Schoenfeld, W. N. Response rate under varying frequency of non-contingent reinforcement. Journal of the Experimental Analysis of Behavior, 1971, 15, 233-236.

Lattal, K. A. Response-reinforcer independence and conventional extinction after fixed-interval and variable-interval schedules. Journal of the Experimental Analysis of Behavior, 1972, 18, 133-140.

Mackintosh, N. J. The psychology of animal learning. New York: Academic Press, 1974.

RACHIIN, H., \& BAUM, W. M. Effects of alternative reinforcement: Does the source matter? Journal of the Experimental Analysis of Behavior, 1972, 18, 231-241.

Rescorla, R. A., \& SkUCY, J. C. Effects of response-independent reinforcers during extinction. Journal of Comparative and Physiological.Psychology, 1969, 67, 381-389.

SKINNER, B. F. The behavior of organisms. New York: Appleton-Century-Crofts, 1938.

Staddon, J. E. R., \& AYres, S. L. Sequential and temporal properties of behavior induced by a schedule of periodic food delivery. Behaviour, 1975, 54, 26-49.

WELKeR, R. L., \& MCAUley, K. Reductions in resistance to extinction and spontaneous recovery as a function of changes in transportational and contextual stimuli. Animal Learning \& Behavior, in press.

ZeILER, M. D. Positive reinforcement and the elimination of reinforced responses. Journal of the Experimental Analysis of Behavior, 1976, 26, 37-44.

(Received for publication September 6, 1978.) 